\title{
Effect of Sudden Water Release on the Reservoir Free Outflow Hydrograph
}

\author{
Vujica M. Yevdjevich ${ }^{2}$
}

(July 9, 1959)

\begin{abstract}
The free outflow hydrograph is studied in the case of a sudden water release from a reservoir. The outflow hydrograph is called free when it is not affected by the tailwater levels. Both the effect of the steep negative wave, created by sudden water release, and the effect of the flow resistance are analyzed through the use of a fictitious inflow hydrograph. The water accelerated by the steep negative wave movement, whether or not the flow resistance is taken into account, gives this fictitious hydrograph. The procedures are given for the computation of the wave celerities and the new water velocities along the reservoir, for the computation of wavefront heights and the maximum outflow discharges, and for the determination of the fictitious and total inflow hydrograph. The examples are given for the pyramidal reservoirs of rectangular and parabolic cross sections. A general procedure for the determination of the reservoir free outflow hydrograph in an approximate form is derived.
\end{abstract}

\section{Introduction}

\subsection{Definition of the Free Outflow Hydrograph}

If the outflow hydrograph from a reservoir is unaffected by the tailwater levels or if such influence is negligible, it will be called the free outflow hydrograph. In that case both the outflow hydrograph and the downstream propagation of the wave created by the sudden release of water can be analyzed separately, first the hydrograph and then the wave propagation.

The reservoir free outflow hydrograph in the case of sudden water release depends on: $(a)$ The area and shape of the outlet; (b) the type of flow (free surface flow or flow under pressure); (c) the shape characteristics of water body (storage function, cross sections, etc); $(d)$ the inflow hydrograph; $(e)$ the time interval in which a full opening of the outlet is created; $(f)$ the movement of negative waves, especially of steep negative waves; $(g)$ the resistance to flow along the reservoir.

The free outflow hydrograph can be approximated by taking into consideration only the most influential of these factors, $(a)$ to $(d)$. Any classical reservoir routing procedure will suffice for the computation. The analytical solutions are possible in cases when the outflow rating curve, the storage function and the inflow hydrograph are given and can be approximated by simple expressions mathematically tractable [3]. ${ }^{3}$ In that case the opening time is assumed as zero and the effects of steep negative wave movement and of flow resistance are neglected, assuming that at the very moment of fast opening the water in the reservoir was instantaneously ac-

1 This work was prepared under a National Bureau of Standards contract with The American University, with the sponsorship of the Corps of Engineers, Army

Map Service.
2 U.S. Geological Survey, Washington 25, D.C

3 Figures in brackets indicate the literature references at the end of this paper. celerated to give maximum discharge. This assumption is the source of the errors in the shape of the free hydrograph, if the steep negative wave is sufficiently high, as when the opening is large in comparison with the reservoir cross sections.

\subsection{Purpose of the Study}

This study seeks the development of a simple procedure for the computation of free outflow hydrograph, when the movement of the steep negative wave and the flow resistance are taken into consideration. The procedure must be suitable for use with the available data, to the accuracy demanded of the resulting hydrographs, and it must require a reasonable computation time.

\subsection{Scope of the Study}

This study will investigate the influence of the steep negative wave and of flow resistance on the shape of the free outflow hydrograph. The knowledge of the shape of reservoir (cross sections) and of its roughness coefficients is needed. Since the opening time is very short in comparison with the outflow time of the major bulk of water, it can be taken as nearly zero.

\subsection{Basic Approach to the Problem}

The shape of the free outflow hydrograph, computed by neglecting the effect of the steep negative wave and of flow resistance, is to be considered as the first approximation of the true outflow hydrograph. This first shape is sufficiently accurate for small negative wave heights in many practical cases, because the accuracy of background data, the routing procedure and the accuracy demanded do not justify more precise computation. The first approximate shape of the free outflow hydrograph is thus con- 
ceived as a gradual emptying of the body of water, starting from already accelerated water [3]. If the neglected factors were taken into consideration and if more data about the reservoir were available, the subsequent approximations would be closer to the true hydrograph.

Two approaches can be used in the computation of the subsequent approximations of the true outflow hydrograph: (1) To develop a special procedure for computing the hydrograph directly from all given data and (2) to correct the previous approximate hydrograph by introducing the effects of new factors. In the second approach the effects are represented by a new fictitious inflow hydrograph. The variables are time, the starting reservoir level, the characteristics of the steep negative wave, the length of reservoir, the flow resistance, etc.

The new approximation of the true hydrograph can be computed from the first approximation by simple transformation using the fictitious function of inflow. Next, the sums of the real inflow hydrograph and fictitious inflow hydrograph can be made. The new inflow hydrographs can be applied directly for the computation of the other approximations of the true hydrograph. This procedure will be used in this study.

\subsection{Effect of the Steep Negative Wave and of Flow Resistance on the Free Outflow Hydrograph}

The outflow from a reservoir in the case of sudden water release does create a steep negative wave which travels upstream along the reservoir.

Every increase of outflow discharge also increases the head losses along the body of water.

When a steep negative wave is created, its movement is influenced by:

(a) The change of the wave area $A_{1}$ and of its shape $\left(A_{1}\right.$ is the vertical projection of the wavefront) and the change of the total cross section area $A$ or of the area under the wave $\left(A-A_{1}\right)$, figure 1 , which change the propagation celerity; $(b)$ the change of the direction and the width of reservoir, of the

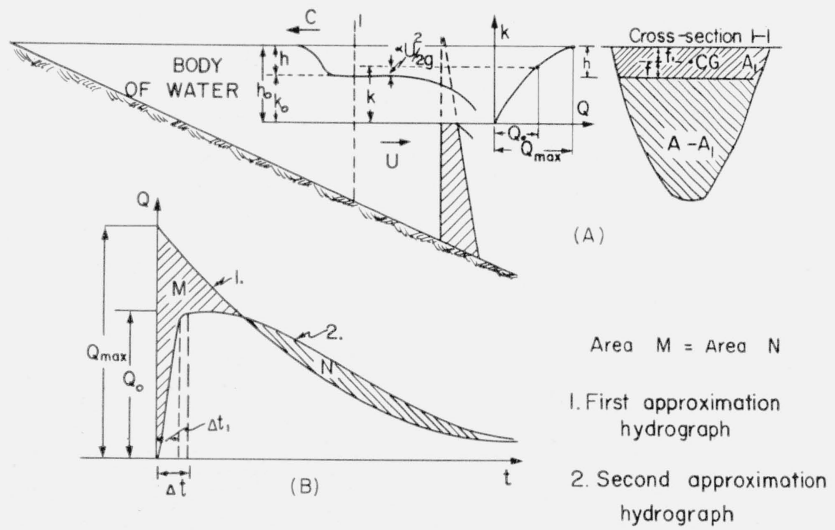

FIGURE 1. Reservoir outflow hydrograph in case of sudden water release.

(A) Schematic reservoir with negative wave movement; (B) two outflow hydrographs: 1-hydrograph with already accelerated water to $O_{\mathrm{max}}$ at the moment of release; 2-hydrograph greatly influenced by the steep negative wave.

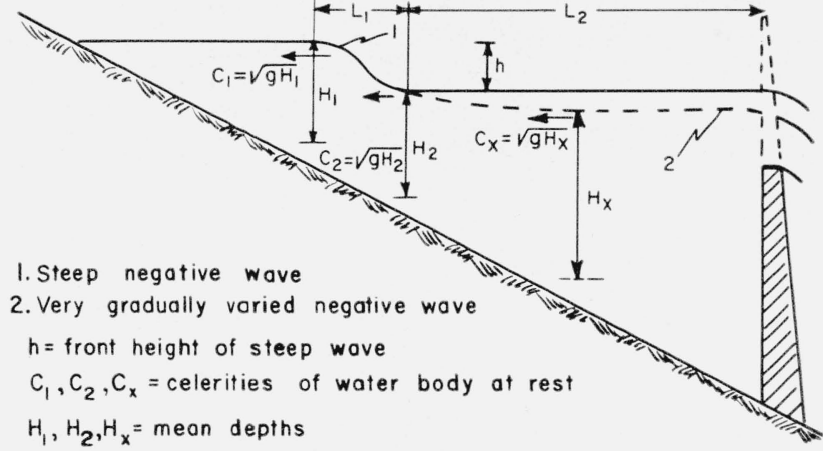

Figure 2. Two types of negative waves in case of sudden water release.

Steep negative wave and long negative wave very gradually varied.

shape and the roughness of its shores, which cause and affect the reflection and the refraction of the wave; $(c)$ the resistance to the acceleration of water due to the movement of the wave, and the resistance of the accelerated water moving downstream between the wavefront and the outlet, which decrease the volume and the kinetic energy of the accelerated water. The flow resistance decreases the outflow discharges in the first part of the hydrograph and increases them in its tail part.

As the propagation celerity of the negative wave gradually decreases in the upstream direction, because of the gradual decrease of mean depth in a reservoir with sloping bottom, the accelerated discharge per unit of time thus correspondingly decreases. As the level at the outlet would be higher at any previous moment than the level corresponding: to the accelerated arriving discharge at the next moment, a new level adaptation must be made to satisfy the continuity equation by a lowering of the level at the outlet, which causes the additional acceleration of water in the reservoir. The volume of water involved in this lowering is taken from surface layers of the body of water between the outlet and the steep negative wave. Therefore, a continuous propagation of small negative waves, changing very gradually, takes place from the outlet upstream. For each time unit a small drop in level at the outlet is created, which propagates upstream with the celerity $C=\sqrt{g H_{x}}$ where $H_{x}$ is the mean depth (fig. 2). As $\mathrm{H}_{x}$ near the steep negative wave is lower than the mean depth of the lowest part of steep negative wave, the small waves can not reach the steep wave. As the process is continuous, the wave movement can be conceived as a group of very small waves making a new long wave, very gradually changing (fig. 2), with $H_{x}$ decreasing.

\subsection{Approximations of the Free Outflow Hydrograph}

The combination of the movement of two types of negative waves, of a very steep wave (depression) and of a very long wave corresponding to gradually varied unsteady flow (with quite opposite characteristics), the complex reflections and refractions 
of waves in a natural body of water, and the resistance to the flow make the exact computation of the free outflow hydrograph very complicated. Therefore, the solutions must be obtained in approximations of a different degree of accuracy. The first approximation neglected the movement of negative waves and the flow resistance (as in [3]); the second approximation will include the addition of the steep wave effect; the third approximation will take account of the additional effect of flow resistance. These two approximations are treated in the subsequent part of this paper. Further approximations can be considered when practically feasible.

\section{Effect of the Steep Negative Wave on the Free Outflow Hydrograph}

\subsection{General Asssumptions}

As the breach of a dam or an accidental opening of some types of large gates are relatively fast processes (a matter of few seconds), the negative wave created by sudden water release is a steep wave traveling upstream. Quite different is the case when the opening of outlets is very slow, the outflow rating curve changing with time as the opening becomes larger. $Q$ is a function of opening dimensions and of time. This type of opening will not be studied here.

The steep negative wave undergoes three types of changes as it travels upstream: (a) Reflections and refractions; $(b)$ decrease of propagation celerity due to the change of cross section; and (c) loss of energy due to the resistance.

The reflection and refraction of the steep negative wave are complex because of the factors which essentially change continuously along the reservoir. The process is, however, somewhat less complex in channels of nearly regular shape. The effects of the reflection and refraction of the steep negative wave will be assumed in the second and third approximations as negligible for the valley reservoirs.

The effect of the negative wave ends when its propagation celerity $C$ in water at rest equals the mean flow velocity $V$ already existent $(C=V)$, or when the wave arrives at the shallow regions, if water is in rest during the wave movement.

The effect of resistance will be neglected in the second approximation, which is justified especially for large bodies of water and moderate heights of steep waves. It will be considered in the third approximation (sec. 3 of this paper).

The main factor in this second approximation is the additional influence of the existence and movement of steep negative wave.

The flow through reservoirs or lakes, where the mean velocities are relatively low and the cross sections change gradually, is a gradually varied flow, either steady or unsteady.

Before the analysis of the effect of the steep negative wave on the outflow hydrograph is begun, two problems will be studied: (1) The propagation of the wave along the water body and (2) the steep wave height and the outflow discharge shortly after the sudden water release.

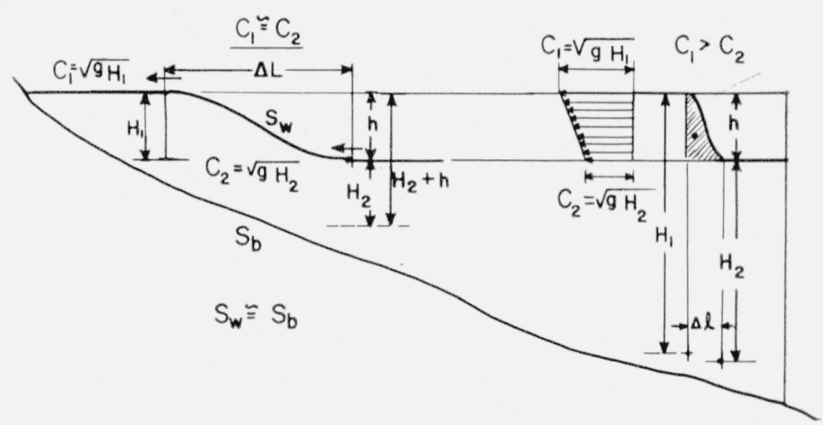

Figure 3. Schematic representation of the transformation on the front shape of steep negative wave, due to different celerities of wave elements.

The shape of a steep negative wave moving along the body of water is not stable (fig. 3). The reason is that the parts of wavefront travel with different celerities. The steepness of the wave decreases with the distance traveled, and the final shape will be obtained when the slope of the wavefront is essentially equal to the slope of the bottom. Because of these differences in celerities of different points of the wave, it is necessary always to define $C$ (as that of the highest point, of the lowest point, of the center of gravity, of the mean height of wave, etc.). The approximations in this study deal with the schematic negative wave, considered as a discontinuous steep wave.

\subsection{Mean Celerity of the Steep Negative Wave in a Cross Section}

The steep negative wave arrives at a cross section of the body of water, $X$ distance from the outlet, at time $t$ from the opening time, with wavefront height $h$. The celerity of the wave in water at rest is $C$ at $X$. The mean velocity of water because of inflow is $V$. The cross section characteristics are (fig. 1): Area $A$ before the wave arrival; area $A_{1}$ of the wavefront height; distance $f$ of the center of gravity $C G$ of area $A_{1}$ from the new level (or from the starting level $f_{1}=h-f$ ); area $A-A_{1}$, after the wave has passed. The effect of the bottom slope is indirectly introduced by the change of $A, A_{1}$, $f, f_{1}$, and $A-A_{1}$.

Two equations, continuity and momentum, give the celerity of the steep negative wave and the increase of the mean water velocity in a cross section of reservoir, due to the acceleration caused by the wave movement, as functions of section characteristics $[1,2]$. For a small time interval $d t$ the celerity can be considered constant in the vicinity of the cross section at $X$, regardless of the bottom slope which has little influence on the cross section characteristics for short path length (fig. 4).

The steep negative wave travels upstream a distance $(C-V) d t$ during the time $d t$ (fig. 4), and the water travels downstream the distance $(V+U) d t$. An observer, traveling at the section $X$ with the velocity $V$ downstream, after time $d t$, will be at the section $X-V d t$, or a distance $V d t$ downstream. To him the distance traveled by the wave in the 


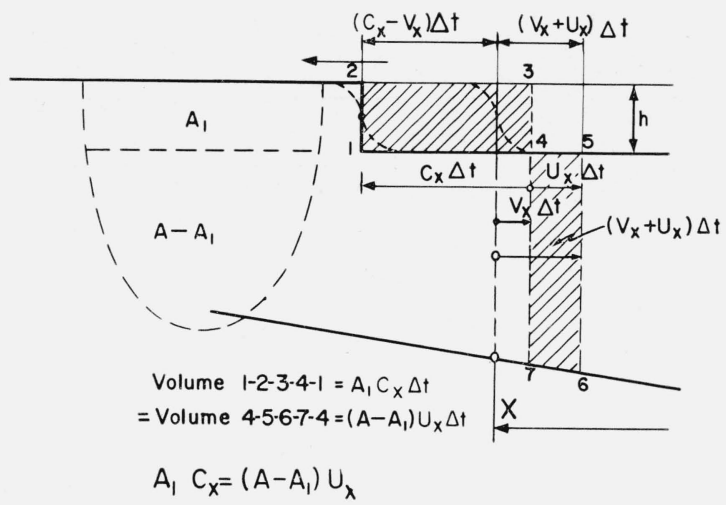

FIgure 4. Scheme for the derivation of continuity equation in case of movement of steep negative waves.

upstream direction is $C d t$ and the distance traveled by the water downstream is $U d t$.

The continuity equation gives for the observer (or also in case $V=0$ )

$$
A_{1} C d t=\left(A-A_{1}\right) U d t,
$$

so that generally for any $X$

$$
C=U\left(\frac{A}{A_{1}}-1\right),
$$

where $C$ is the propagation celerity of the steep negative wave in water at rest and $C-V$ is the propagation celerity in moving water with velocity $V(V<C)$. Equation (1) can be obtained also by equating the total water discharge entering the small volume $A d x$, to the discharge leaving that volume

$$
V A+(C-V) A_{1}=(V+U)\left(A-A_{1}\right) .
$$

For the momentum equation, it is assumed that the acceleration of water from the velocity $V$ to the velocity $V+U$ of the volume of water $\left(\ddot{A}-A_{1}\right) C d t$, in time $d t$ and at the section $X$ (figs. 4 and 5 ) is made without loss of energy.

The volume element $\left(A-A_{1}\right) C d t$, which is accelerated from $V$ to the velocity $V+U$ between times $t$ and $t+d t$, has a momentum $\left(A-A_{1}\right) C U d t$ and a rate of change of momentum $\left(A-A_{1}\right) C U$. The force $F$ which creates this change is the difference of pressure on the areas $A$ and $A-A_{1}$, (fig. 5), $F=g h\left(A-A_{1}\right)+g f A$, with $f$ already defined.

This force is equal to the rate of change of momentum, so that

$$
C U=g h+g f \frac{A_{1}}{A-A_{1}} .
$$

The celerity $C$ at any section in water at rest, after using eq (1), is

$$
C=\sqrt{g h(a-1)+g f},
$$

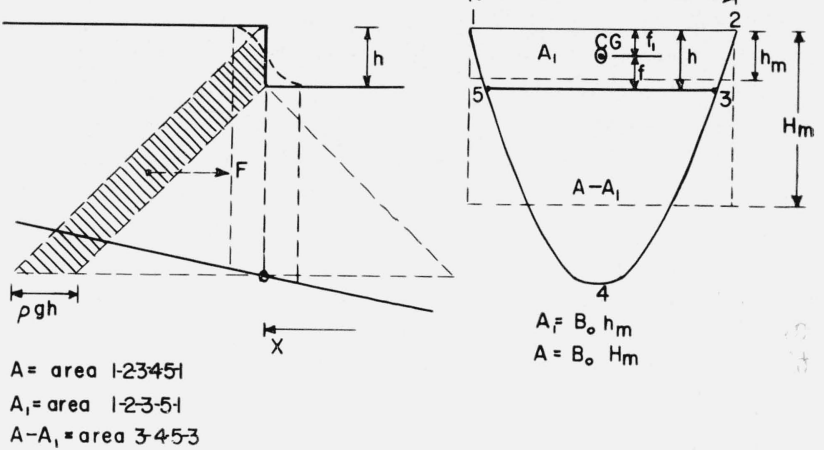

Figure 5. Scheme for the derivation of momentum equation in case of steep negative wave movements.

where $a=A / A_{1}$. The ratio $a>1$, and theoretically can be between the limits 1 and infinity. If $h$ increases, $(a-1)$ decreases faster than $h$ and $f$, so that the mean celerity of the wavefront decreases with the increase of $h$. With the mean depths of areas $A$ and $A_{1}, H_{m}=A / B_{0}$ and $h_{m}=A_{1} / B_{0}$ (fig. 5), with $B_{0}$ the width at the surface level, and putting $f_{1}=h-f$, eq (2) becomes

$$
C=\sqrt{g\left(H_{m} \frac{h}{h_{m}}-f_{1}\right)} .
$$

When $h / h m$ is nearly 1 and $f_{1}$ can be neglected in comparison with $H_{m}$ (for small values of $h$ ) then

$$
C=\sqrt{g H_{m}}
$$

which is the known expression for the propagation of small surface disturbances in shallow water, with the mean depth $H_{m}$.

The increase of the mean water velocity is, therefore, from eqs (1) and (2)

$$
U=\frac{1}{a-1} \sqrt{g h(a-1)+g f},
$$

or from eqs (1) and (3)

$$
U=\frac{h_{m}}{H_{m}-h_{m}} \sqrt{g\left(H_{m} \frac{h}{h_{m}}-f_{1}\right)},
$$

which approaches zero when $h$ approaches zero.

The discharge $P_{w}=U\left(A-A_{1}\right)$ is the accelerated discharge by the movement of the steep negative wave, which will be considered as the fictitious inflow in the reservoir.

As $H_{m}, h_{m}$, and $f_{1}$ are functions of cross section characteristics and of height of wavefront $h$, both $C$ and $U$ are functions of those parameters. The cross section characteristics are functions of the water level. For a given section, level, and height $h$ the values $C$ and $U$ are uniquely determined. 
2.3. Computation of the Initial Front Height of Steep Negative Wave and of the Maximum Outflow Discharge

If an opening in a reservoir is created with constant area and shape, its hydraulic characteristics are defined by the outflow rating curve, generally of the type $Q=b k^{r}$, where $b$ and $r$ depend on the area, shape and position of the opening, as well as on the type of flow, with $k$ the total energy head above the level of the outflow zero (fig. 1). The opening height, as the difference between the level at the moment of opening and the level of outflow zero, is $h_{0}$. It is the sum of two heights: $h$, the front height of the steep negative wave and $k_{0}$, the difference of the level just upstream from the outlet (where the depression due to the outflow is negligible) and the level of outflow zero, so that $h_{0}=h+k_{0}$. The energy head is

$$
k=k_{0}+\frac{\alpha(V+U)^{2}}{2 g},
$$

where $V$ is the velocity near the opening before it is created and $\alpha$ is the kinetic energy correction factor.

For the small time interval, $\Delta t$, after the opening, the water flowing through the opening is the sum of the inflow discharge $P=A V$ and the accelerated discharge due to the moving steep negative wave. The interval $\Delta t$ must be sufficiently long for the negative wave to develop but not so long as to increase the outflow by the further significant drop in water level of the stretch of reservoir between the outlet and the steep negative wavefront.

The continuity equation between supply and outflow gives

$$
Q_{0}=A V+A_{1}(C-V)=b k^{r},
$$

which can be written as [4, p. 213, without last term]

$$
Q_{0}=A V+A_{1}(C-V)=b\left(k_{0}+\frac{\alpha(V+U)^{2}}{2 g}\right)^{r} .
$$

For small value of velocity head in comparison with $k_{0}$, eq (9) can be expressed in the approximate form

$$
Q_{0}=b k^{r}=b k_{0}^{r}\left[1+\frac{r \alpha(V+U)^{2}}{2 g k_{0}}\right] .
$$

The second term of $\left[4\right.$, p. 213] in form of $\left(V_{0}^{2} / 2 g\right)^{3 / 2}$, which takes here the form

$$
-b\left[\frac{\alpha(V+U)^{2}}{2 g}\right]^{r}
$$

and should be added on the right side of eq (9), can be neglected for reservoir outflows in comparison with the first term. The celerity $C$ is given by eq (2) or (3), and velocity $U$ by eq (5) or $(6)$.

For given $h_{0}, b$, and $r$ (values or functions dependent only on the outlet characteristics), and given $V$ and $\alpha$, the magnitudes $A_{1}, C, U$, and $k_{0}=h_{0}-h$ are functions of $h$ and of the cross section characteristics near the outflow, at distance $\Delta X=(C-V) \Delta t$. When the cross section at distance $\Delta X$ from the outlet is selected, eq (9) enables the computation of the value $h$. As the discharges around the maximum discharge change slowly (fig. 1), the selection of $\Delta X$ is easier. It is, therefore, sufficient to select an average cross section some hundred meters upstream from the outlet. For given $h$, the values $k_{0}$ and $U$, or $A_{1}$ and $C$ can be obtained, so that eq (9) also gives $Q_{0}$, the discharge outflow at the time $\Delta t$ (which is the maximum outflow if $\Delta X$ is well selected).

The computation of $h$ is to be made by successive approximations until eq (9) is satisfied for the computed $h$. Numerical or graphical procedures are feasible for that computation.

A simple graphical procedure for the determination of $h, Q_{0}$ and downstream tailwater level $H_{d}$ is shown in figure 6. Three curves as the functions of $h$ and independent of the height $h_{0}$ of the opening are first plotted:

$$
\begin{gathered}
Q_{1}=A_{1}(C-V)+A V, \\
\Delta k_{0}=\frac{\alpha(V+U)^{2}}{2 g},
\end{gathered}
$$

and

$$
Q_{3}=f\left(H_{d}\right),
$$

where $Q_{3}$ is the rating curve of the tailwater channel. For the given opening height $h_{0}$ the fourth curve $Q_{2}=b k^{r}$ is plotted. For some types of outlets, the coefficient $b$ is a function of $k_{0} /\left(H_{0}-h_{0}\right)$ and of some other parameters, so that approximate values must be first introduced. For convenience the velocity head $\Delta k_{0}$ is plotted with the same scale as $h_{0}, h, k$, and $k_{0}$. For an assumed value $h$ or $k_{0}$ (point 1 ) the discharge $Q_{1}=A_{1}(C-U)+A V$ is obtained (curve 1, point 3 ), as well as $\Delta k_{0}$ (curve 3 , point 2 ). By adding $\Delta k_{0}$ to $k_{0}$ (point 4) the value $k$ is obtained, and $Q_{2}=b k^{r}$ (curve 2, point 5). The adjustments have to be made for $h$ until $Q_{1}=Q_{2}$. If $Q_{1}=Q_{2}$, the value of $h$ is obtained. From that value the discharge

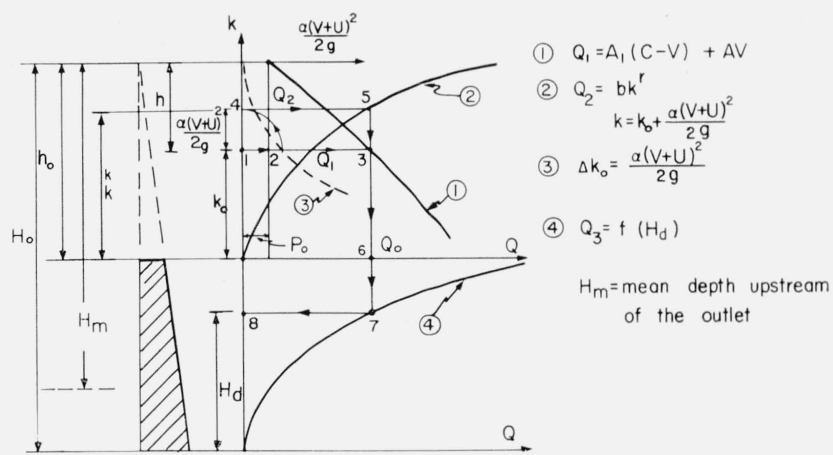

FIGURE 6. Graphic procedure for the determination of front height of steep negative wave, of maximum discharge, and of maximum tailwater level.

1 -accelerated discharge plus inflow $P_{0}$ as function of wavefront height; 2 outflow rating curve; 3 -total velocity head as the function of wavefront height; 4 -tailwater rating curve. 
$Q_{0}$ is determined (point 6). For a given $Q_{0}$ (curve 4, point 7 ) the maximum downstream level $H_{d}$ (point 8) is obtained. When $H_{d} \leq \beta\left(H_{0}-h_{0}\right)$, the outflow is uninfluenced by tailwater leval. The coefficient $\beta \geq 1$ is a correction factor which takes into account that, due to the effect of kinetic energy of outflow water, the tailwater levels somewhat higher than $\left(H_{0}-h_{0}\right)$ do not influence the outflow.

\subsection{Relation of the Height of Steep Negative Waves to the Opening Height of a Rectangular Outlet}

For the given opening, the values $h$ and $Q_{0}$ depend on the shape of three curves, 1,2, and 3 of figure 6 . The accuracy of curve $2\left(Q=b k^{r}\right)$ depends on the accuracy of the dimensionless outflow coefficient $m$ which enters into the expression for the coefficient $b$.

The case of a reservoir, with water at rest, of approximately rectangular cross section $A=B_{0} H_{m}$ near the outlet will be treated here. The bottom of small length of reservoir $\Delta X$ can be assumed horizontal, especially under natural conditions with deposited sediments. The simple case of a rectangular sharp-edged opening will be analyzed here as an example, with area $B h_{0}$, where $h_{0}$ is much smaller than $H_{m}$ (fig. 6), and $B \leq B_{0}$. Equation (9) will be used, and according to [4] and [5], with different symbols used here,

$$
m=\frac{Q}{B h \sqrt{g h}}=\frac{Q}{B_{0} \sqrt{g} h^{3 / 2}} \cdot \frac{B_{0}}{B}=\frac{b}{B_{0} \sqrt{g}} \cdot \frac{B_{0}}{B},
$$

or

$$
m=\frac{B_{0}}{B} \cdot f\left(\frac{k_{0}}{H_{m}-h_{0}}, R, W\right)
$$

with $k_{0}=h_{0}-h$ and $R$ and $W$ the Reynolds and Weber numbers, respectively.

In this expression $B_{0}$ is taken in the denominator instead of the usual $B$, so that the right side of eq. (10) is multiplied by $B_{0} / B$, apart from the contraction effect dependent on $B / B_{0}$, in order to facilitate the solution of eq (9), regardless of the changing width $B$ of the opening.

Putting $h_{0} / H_{m}=s$ and $h / H_{m}=p$, the ratio $h / h_{0}=p / s$, of the wave height $h$ and the opening height $h_{0}$ will be related to $s$. For the rectangular sharp-edged opening

$$
m=\frac{B_{0}}{B} f\left(\frac{B}{B_{0}}, \frac{s-p}{1-s}, R, W\right)
$$

where $m$ is $B_{0} / B$ times higher than the usual coefficient $m$. Other functions of $m$ for other shapes of outlet have to be used, depending on the outlet.

The coefficient $m$ can be thus expressed as a function of dimensionless numbers, so that for each outlet and its characteristics the special relation of eq (11) or of other type can be obtained. For given $B, B_{0}$, $H_{m}$ and $h_{0}$, the coefficient $m$ can be expressed as a function of $h$, or of $p=h / H_{m}$.

Equation (9) under the above assumptions and in terms of the dimensionless numbers introduced here can be written as

$$
s=p+\frac{p^{2 / 3}}{m^{2 / 3}}\left(1-\frac{p}{2}\right)^{1 / 3}-\frac{\alpha}{4} \frac{p^{2}(2-p)}{(1-p)^{2}} .
$$

If the function of eq (11) is introduced in eq (12), the relationship $p=f(s)$ is obtained. For the practical solution it is suitable to take $m$ as a parameter and to develop a family of curves for eq (12). Figure 7 gives this family of $p=f(s)$ for $m=0.25,0.50,0.75$, $1.00,1.25$, and 1.50 , and figure 8 gives $p / s=f(s)$ for $m=0.25,0.50,0.75$, and 1.00 . The families of curves $m=f(p)$ or $m=f\left(\frac{p}{s}\right)$ for given values $s$ are more convenient to use. Both are given in figures 7 and 8 as dashed lines. For these curves $\alpha=1.05$ was taken as the most applicable constant value.

When eq (11) is determined for a particular case of rectangular opening, with $s=h_{0} / H_{m}$ known, the intersection of the curve of eq (11) and the curve $m=f\left(h / H_{m}\right)=f(p)$ or $m=f\left(h / h_{0}\right)=f(p / s)$ from figure 7 or 8 for that $s$, gives the solution either for $p=h / H_{m}$ or $p / s=h / h_{0}$.

If the velocity $V$ in the body of water near the outlet, before the sudden release of additional water, can not be neglected in comparison with the new velocity $(V+U)$, it must be introduced in dimensionless form in eq. (12). In that case, for $w=$ $V_{0} / \sqrt{g H_{m}}=V_{0} / C_{m}$, with $C_{m}=\sqrt{g H_{m}}$, and $V_{0}=P / B_{0} H_{m}$,

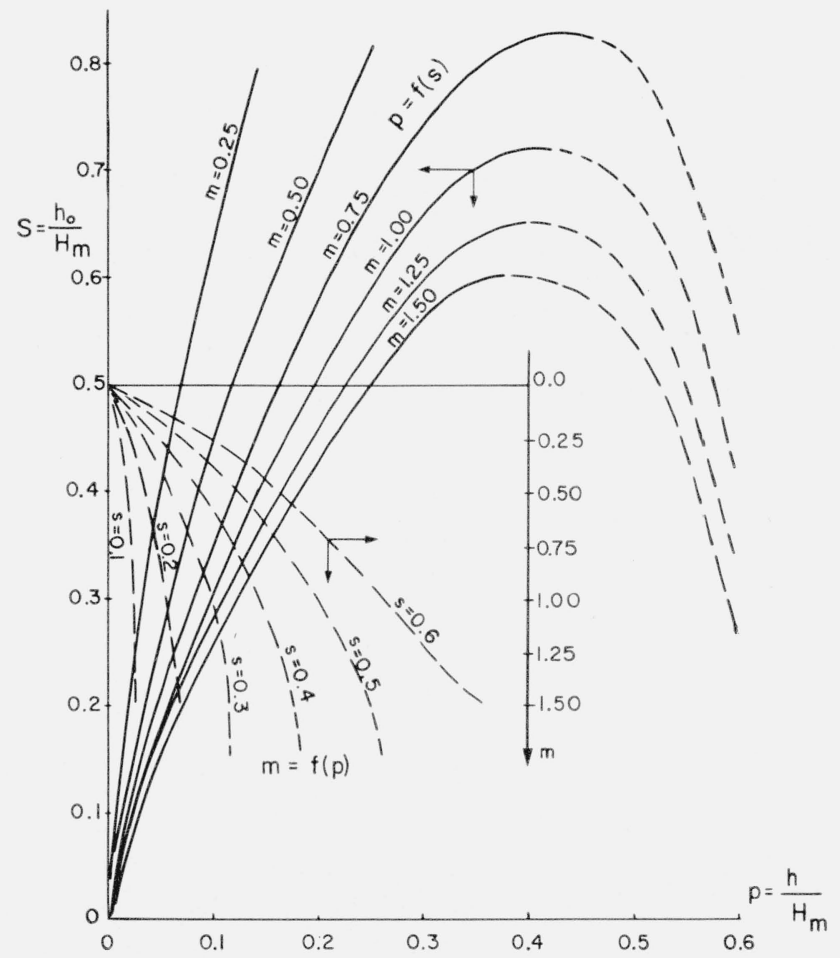

FiguRE 7. Relation of the relative height of wavefront $\left(p=h / H_{m}\right)$ to relative opening height $\left(s=h_{0} / H_{m}\right)$ where $H_{m}$ is mean depth of reservoir near outlet, with the outflow coefficient $m$ as parameter, for rectangular opening; the second family is the relation of $m$ and $p$ with $s$ as a parameter. 


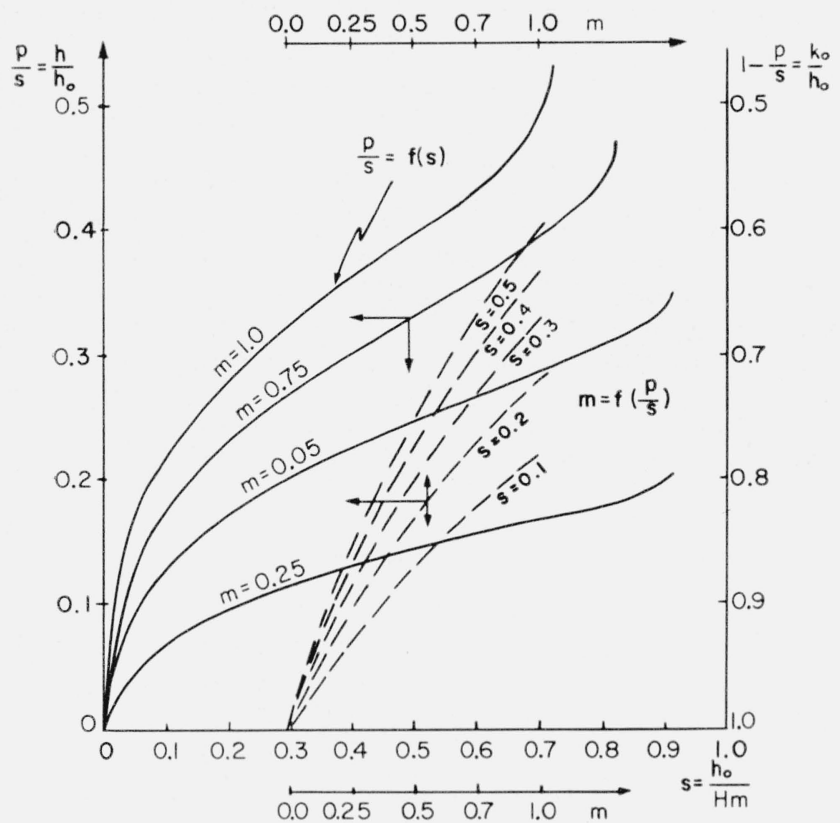

Figure 8. Relation of the ratio of wavefront height and opening height $\left(h / h_{0}\right)$ to relative opening height $\left(h_{0} / H_{m}\right)$ with outflow coefficient $m$ as parameter for rectangular opening; dashed lines represent the relation of $m$ and $p / s$ with $s$ as parameter.

$s=p+\left[\frac{p}{m}\left(1-\frac{p}{2}\right)^{1 / 2}+\frac{w}{m}\right]^{2 / 3}-\frac{\alpha}{2}\left[w+\frac{p}{1-p} \sqrt{1-\frac{p}{2}}\right]^{2}$.

For each given value of $w$ a family of curves $m=f\left(h / H_{m}\right)$ or $m=f\left(h / h_{0}\right)$ can be developed.

Equations (12) and (13) in parametric form can be developed for any regular-shaped outlets (rectangular, triangular, semicircular, circular, trapezoidal, parabolic, semielliptic, etc.). With $s$, or with $s$ and $w$, as parameters, the families of curves $m=f\left(h / h_{0}\right)$ or $m=f\left(h / H_{m}\right)$ can be developed once for all cases. With these families available, the determined real function $m=f\left(h / h_{0}\right)$ for a particular outlet of given regular shape will be sufficient for the determination of the height $h$ of the steep negative wave, and of the maximum discharge of outflow hydrograph $Q_{0}$ by using eq (8). The nomographs can also be developed for the relations in eqs (12) and (13).

If $m$ is selected to be only the function of $h$, then eq (12) would give a stable relationship between $p$ and $s$, or eq (13) would give a family of curves with $w$ as parameter.

\subsection{Procedure for the Computation of a Free Outflow Hydrograph}

In the case of no inflow into the reservoir, the celerity $C$ depends on the wave height $h$ and on the depth $H_{0}$ of reservoir at the outlet before the sudden water release. The celerity is, therefore, the general function

$$
C=f\left(x, \frac{h}{H}, \frac{H}{H_{0}}\right)
$$

where $x=1-X / L$ is the relative distance of a cross section from the upstream end of reservoir, $X$ is the distance from the outlet, $L$ is the length of reservoir, $H$ is any possible reservoir depth at the outlet, $H_{0}$ is the maximum operational depth, $h$ the height of the steep negative wave.

For a given relative parameter $H / H_{0}$, a family of curves $C=f\left(x, h / H_{0}\right)$, can be plotted.

For a large reservoir, $\mathrm{H}_{0}$ and $h$ will be assumed as the initial or boundary conditions. For a given cross section $x$ the values $A, A_{1}, a, f, C$, and $U$, entering into eqs (2), (3), (5), and (6), are also given.

The general procedure for the second approximation of taking into consideration the effect of the movement of steep negative wave in the case of valley reservoirs is:

(1) To compute the wave height $h$ for given water depth $H$ at the outlet, and to determine the maximum outflow discharge $Q_{0}$, as is shown in subsections 2.3 and 2.4 , eqs $(7)$ to (13), figures 6 to 8 .

(2) To verify that the outflow hydrograph is free, or that the tailwater level is lower than the maximum tailwater level which does not affect the outflow (fig. 6) ;

(3) To compute the celerity $C$ along the body of water out of given cross section characteristics ( $A$, $A_{1}$ and $\left.f\right)$, eqs (2) and (3);

(4) To compute the velocity $V$ along the body of water for the inflow $P$ during the wave movement, if there is an inflow;

(5) To determine the difference $(C-V)$ along the body of water;

(6) To compute the accelerated discharges by steep negative wave movement along the body of water $P_{w 1}=C A_{1}$ with inflow zero, or $P_{w 2}=(C-V) A_{1}$ with inflow $P$;

(7) To determine the time, when the wave arrives at the section $X$ and when the acceleration takes place there, by $t=\int_{X=0}^{X} \frac{d x}{C-V}$, or for $1-\frac{X}{L}=x$ ( $L=$ length of reservoir) by

$$
t=L \int_{x}^{1} \frac{d x}{C-V}
$$

with $t_{0}$ the total time of wave travel from the outlet to the upstream end of reservoir (from $x=1$ to $x=0)$;

(8) To find the relation between the accelerated discharge and the time of acceleration, $P_{w}=f(t)$. For given $x$ the pair of values $P_{w}$ and $t$ gives $P_{w}=f(t)$.

The integration of $P_{w}$ over time from $t=0$ to $t=t_{0}$ gives the volume $S_{h}$ of the reservoir layer $h$, determined simply from the storage function $S=f(H)$, figure 10 , which can serve to check the function $P_{w}=f(t)$. 
If the inflow during time $t_{0}$ cannot be taken as constant (i.e., the natural inflow hydrograph or the release of water from upstream reservoirs are very unsteady), the velocity $V$ at a cross section $x$ is also dependent upon the time as are $C-V, A_{1}$ and $P_{w}$. The inflow can be either a steep or a gradual wave and the determination of $P_{w}=f(t)$ becomes more complex.

If the inflow is in form of a steep positive or negative wave, the procedure is similar to the above given movement of steep negative wave until two waves traveling in opposite directions meet. The resulting waves will then move in both directions.

In the case where the inflow hydrograph $P=f(t)$ is gradually varied, and where the steep negative wave moves faster upstream than the other negative waves created downstream of the steep wave, a resulting new inflow hydrograph $P_{n}=f(t)$, composed of $P=f(t)$ and the accelerated discharges by steep negative wave movement, can be determined either by exact or approximate methods. The position of the wave $P=f(t)$ along the reservoir has to be determined. Therefore, the velocity $V$ for given places and the times at which the steep negative wave arrives at those places must be computed in order to obtain the celerity $C-V$. The celerity is now $C=f\left(x, t, \frac{h}{H}, \frac{H}{H_{0}}\right)$, with a new variable, $t$.

The time $t_{0}$ of wave travel from one end of the reservoir to the other is the factor which will determine whether or not the inflow hydrograph can be replaced by a constant inflow $P_{0}$. Since $t_{0}$ for the majority of valley reservoirs is measured in hours, constant inflow can often be assumed during the movement of steep negative wave.

Due to the neglected factors - resistance to the flow, reflection and refraction of the wavefront and transformation of the wavefront due to different celerities of its parts - the computed function $P_{w}=f(t)$ is an approximation of the true relationship.

The procedure for the second approximation of the free outflow hydrograph would involve the following values and functions:

(1) The outflow head $k=k_{0}+\frac{\alpha(V+U)^{2}}{2 g}$; (2) the outflow rating curve $Q=b k^{r}$; (3) the characteristics of cross sections along the reservoir, and (4) the total inflow function $P_{n}=f(t)$, which gives for each time $t$ the discharge $P_{n}=P+P_{w}$, and for the corresponding $x$, also, the place of inflow $P_{n}$. The use of the two basic de Saint Venant equations for gradually varied unsteady flow will enable the computation of the free outflow hydrograph in cases where the continuity equation alone can not give reliable results.

In valley reservoirs, where the height of the negative wave is not too large, the use of the continuity equation alone, instead of both continuity and momentum, gives an approximate free outflow hydrograph, sufficiently accurate in the light of background data, assumptions and the needed accuracy of results.

The higher the steep negative wave and the inflow $P$, the greater is the velocity $(V+U)$, so that the use of the continuity equation alone will give less accurate results. Since the effect of neglected factors in steep negative wave movement is the highest in shallow waters (upstream part of reservoirs) and lowest in the deep waters (lower part of reservoirs) and since $P_{w}$ is low in the first and high in the second region, the use of only the continuity equation is generally justified. In that case the integration of the storage differential equation (continuity equation) of the type $P-Q=d S / d t$, with $P$ replaced by the new inflow hydrograph $P_{n}=P+P_{w}$, can be made by a numerical, graphical, or any other appropriate procedure to obtain the free outflow hydrograph $Q=f(t)$.

Two simple cases will be taken as examples: $(a) \mathrm{A}$ pyramidal reservoir with rectangular sections $\left(B_{X}\right.$, $H_{X}$ ), with the bottom slope $H_{0} / L$, where $H_{0}$ is its depth at outlet and $L$ reservoir length, and with $B_{0}$ the width of reservoir at the outlet; $(b)$ a parabolic section of the type $B=B_{0} \sqrt{H / H_{0}}$, where $B_{0}$ and $H_{0}$ are width and depth respectively of reservoir at the outlet, $B$ and $H$ any width and depth, with the bottom slope $H_{0} / L$, and $B_{x}$ and $H_{x}$ the surface width and water depth at a cross section $X$ distance from outlet.

\subsection{Rectangular Cross Sections}

Figure 9 gives the simple shape of the reservoir and the resulting functions along it for the inflow $P=0$. The steep negative wave has the front height $h$ with $p=h / H_{0}$, and $x=1-(X / L)$, so that $B_{x} / B_{0}=H_{x} / H_{0}=x$.

The total area of any cross section is $A=x^{2} B_{0} H_{0}$ and the wavefront area $A_{1}=x h B_{0} ; f=h / 2$, so that

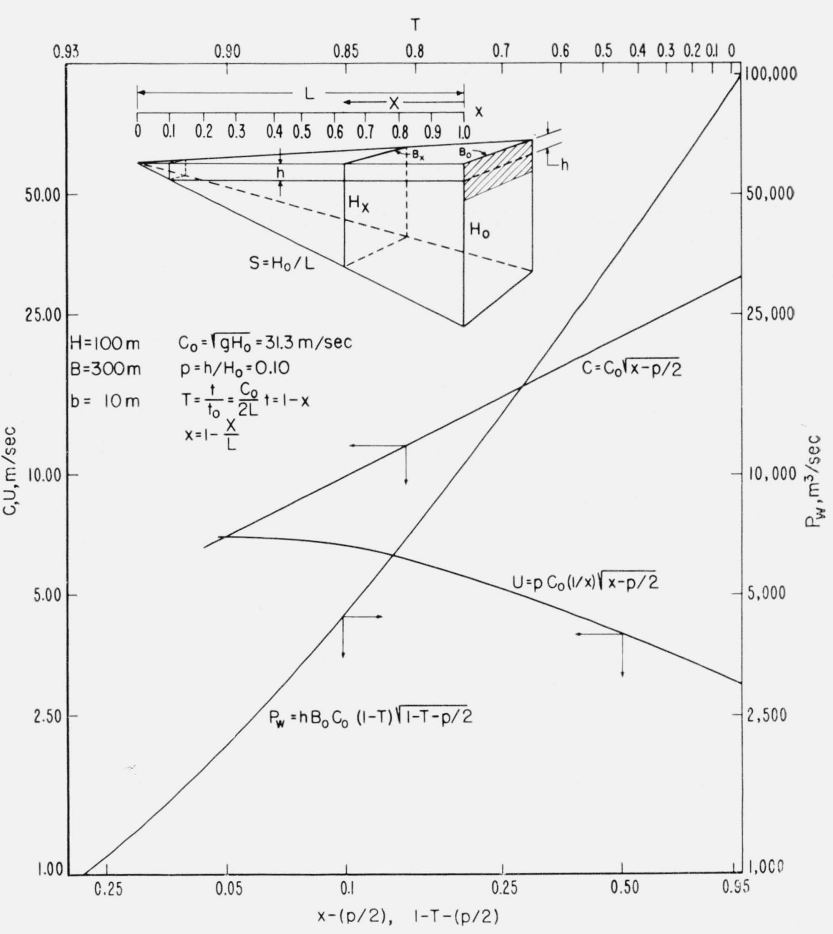

Figure 9. Celerity, velocity of accelerated water, and accelerated discharges along a pyramidal schematic reservoir with rectangular cross sections and rectangular opening of the same width as the reservoir (upper figure). 
from eq. (2)

$$
C=C_{0} \sqrt{x-\frac{p}{2}}
$$

with $C_{0}=\sqrt{g H}_{0}$, the maximum celerity of the uppermost part of steep negative wave near the outlet. In the case of constant inflow $P_{0}$ and $V=P_{0} / A$,

$$
C-V=C_{0} \sqrt{x-\frac{p}{2}}-\frac{V_{0}}{x^{2}},
$$

with $V_{0}=P_{0} / B_{0} H_{0}$ the velocity at the outlet before the sudden water release. The new velocity along the body of water after the wave passes is, according to eq (5),

$$
V+U=\frac{V_{0}}{x^{2}}+C_{0} \frac{p}{x} \sqrt{x-\frac{p}{2}} .
$$

For small values of $x$ eq (16), (17), and (18) have no practical meaning, because $C$ and $U$ are very high, and the resistance plays the major role, so that they should be used for values slightly higher than $p$. The accelerated discharge plus the inflow $P_{0}$ is now $P_{n}=P_{0}+P_{0}$, with

$$
P_{w}=h B_{0}\left(C_{0} x \sqrt{x-\frac{p}{2}}-\frac{V_{0}}{x}\right) .
$$

The travel time from the outlet to a cross section $X$ distance, in the case $V=0$, is

$$
t=\int_{0}^{X} \frac{d X}{C}=L \int_{X}^{1} \frac{d x}{c}=\frac{2 L}{C_{0}}(1-x) .
$$

If $x=0$, the travel time along the reservoir for $V_{0}=0$ is

$$
t_{0}=\frac{2 L}{C_{0}}
$$

and $x=1-\frac{t}{t_{0}}=1-T$, where $T=t / t_{0}$. With the increase of $P_{0}$ the time $t_{0}$ increases and the discharge $P_{w}$ decreases, though $P_{n}=P_{0}+P_{w}$ increases.

The integration of the $t$-function for $V_{0} \neq 0$ in the form

$$
t=L \int_{X}^{1} \frac{x^{2} d x}{C_{0} x^{2} \sqrt{x-\frac{p}{2}}-V_{0}}
$$

is best made numerically.

The functions $C, U$, and $P_{w}$ are given in logarithmic scales in figure 9 for the following values: $H_{0}=100 \mathrm{~m}$; $B_{0}=300 \mathrm{~m} ; h=10 \mathrm{~m} ; C$ and $U$ in $\mathrm{m} / \mathrm{sec}$ and $Q$ in $\mathrm{m}^{3} / \mathrm{sec}$.

\subsection{Parabolic Cross Section}

For given $h$ and $H_{0}$, and two cases of the inflow: $P=0$ and $P=P_{0}$, figure 10 gives the following functions depending on $x=1-(X / L)$ for the parabolic cross sections of the type $B=d \sqrt{H_{0} H}$, where $d=B_{0} / H_{0}$;
$A ; A_{1} ; a=A / A_{1} ; C$ (or $\left.C-V\right) ; U$ (or $\left.U+V\right) ; V=P_{0} / A$; $t=\int_{1}^{x} \frac{d x}{C}\left(\right.$ or $\left.t=\int_{1}^{x} \frac{d x}{C-V}\right) ; P_{1}$ (or $P_{2}$ ), accelerated discharge $P_{w}$ or $P_{n}=P_{0}+A_{1}(C-V)$; and finally $P_{1}$ (or $P_{2}$ ) as a function of time $t_{1}\left(\right.$ or $\left.t_{2}\right)$.

\subsection{Shape of the Hydrograph in the First Moments After Release}

A few moments after the release, at the time interval $\Delta t$, a full steep negative wave of height $h$ is developed (fig. 1). At that moment the outflow is maximum. The approximate solution for the shape of the rising part of the hydrograph after the sudden water release can be obtained as a straight line for an ideal fluid by using the two-dimensional hydrodynamic equations of motion expressed in Lagrangian form [6].

If the straight line rise is assumed for the total rise, the procedure for the determination of the slope of the hydrograph is simple. The total outflow of water from the time of opening to the time $\Delta t$ is approximately the volume of water confined between the outflow section and the section at $\Delta X=(C-V) \Delta t$, where $(C-V)$ is the wave celerity at the beginning, and between the starting depth of water $H$ at the outlet and the new depth $H-h$. This volume is $\Delta S$. If it is taken that the maximum discharge $Q_{0}$ takes place at time $\Delta t_{1}<\Delta t$, instead of at $\Delta t$, the continuity equation gives $1 / 2\left(\Delta t+\Delta t_{1}\right) Q_{0}=\Delta S$, and $\Delta t_{1}$ can be obtained (fig. 1). The mean slope of the straight line for the rising part of the hydrograph is then $Q_{0} / \Delta t_{1}$. For small $h$, the values $\Delta S$ and $\Delta T$ are very small, and the rise of hydrograph can be taken as vertical.

Figure 11 gives an example of the difference between the outflow hydrograph of the first approximation, curve 1 (emptying of the reservoir with the negative wave movement neglected) and the outflow hydrograph of the second approximation, curve 3 (with the steep negative wave taken into consideration, but with the flow resistance neglected) for the Savage River Reservoir near Cumberland, Md. A rectangular opening $(250 \mathrm{~m}$ by $18.5 \mathrm{~m})$ with sudden water release in the case of a full reservoir is assumed. Curve 2 gives the fictitious inflow hydrograph computed by the method given above. Though the total volume of the two hydrographs, 1 and 3 , is the same, the peak discharge in the second approximation is smaller for about one-third.

\section{Effect of Flow Resistance on the Free Outflow Hydrograph}

\subsection{Two Aspects of the Flow Resistance}

The effect of the flow resistance on the free outflow hydrograph depends on the new velocities along the reservoir. The greater the wavefront height $h$, the less the remaining flow area $A-A_{1}$ after the steep negative wave has passed, and the greater the inflow and the roughness coefficient in the reservoir, the greater will be the flow resistance effect. 

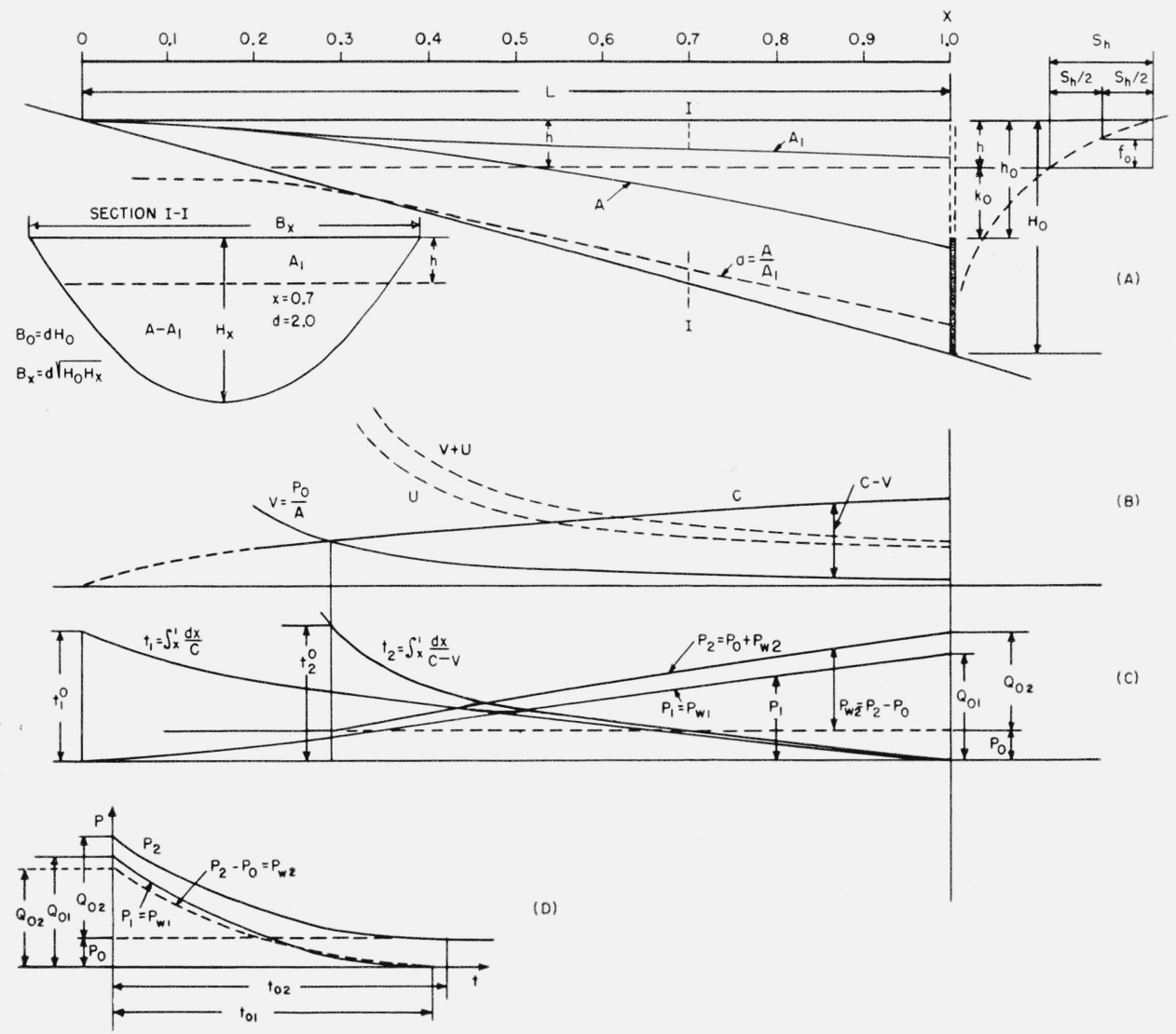

FIGURE 10. Schematic representation of the cross-section characteristics and hydraulic magnitudes along a pyramidal reservoir with parabolic cross sections.

(A) reservoir with areas $A$ and $A_{1}$ and their ratio $a=A / A_{1}$ along the reservoir with a cross section at left and storage function of the reservoir at right; (B) celerity $C$ and velocity $U$ of accelerated water, due to the steep wave movement function of the reservoir at right; (B) celerity $C$ and velocity $U$ of accelerated water, due to the steep wave movement along the reservoir in case where water is at rest, and celerity $C-V$ and velocity $V=P_{0} / A$ and $V+U$ along the reservoir in case where inflow of water in reservoir is $P_{0}$; (C) accelerated discharge and times at which the acceleration take place along the reservoir for two cases: inflow is $\mathrm{O}$ and inflow is $P_{0} ;(1)$ fictitious and total inflow hydrographs for reservoir in two cases: inflow is $\mathrm{O}$ and inflow is $P_{0}$ (third-dashed-curve gives fictitious hydrograph in case inflow $P=0$ ).

Two aspects of flow resistance can be taken into consideration: (1) Head losses along the reservoir, which can be taken into account by introducing the friction slope into the second or dynamic de Saint Venant equation for unsteady flow gradually varied; and (2) decreasing of front height of the steep negative wave, because of the downstream increase of level due to the resistance head as the wave progresses upstream. Hence, the effective potential energy of wave (energy to be transformed into kinetic energy of accelerated water) will be smaller than the total energy corresponding to the initial height $h$.

The first aspect of resistance can be taken into account when the routing procedure is based on the two de Saint Venant equations. It is, however, possible to take into consideration the resistance effect on steep negative wave movement when either the continuity equation of the type $P-Q=d S / d t$ or both the continuity and dynamic equations are used for the routing. The effect of flow resistance on the fictitious inflow hydrograph, caused by the steep negative wave movement, will be dealt with in this investigation.

\subsection{Effect of the Flow Resistance on the Steep Nega- tive Wave}

If a cross section at distance $X$ from the outlet has the following values determined: $A, A_{1}, f, C, V, U$, and if the resistance effect is introduced, the height $h$ will decrease along the reservoir, with the slope

$$
\frac{d h}{d x}=\frac{n^{2}(V+U)^{2}}{R^{4 / 3}},
$$

where the Manning formula for the flow resistance is used in metric system, where $n$ is the roughness coefficient and $R$ the hydraulic radius of area $\left(A-A_{1}\right)$. At the distance $x=1-(X / L)$ the new wave height $h_{1}$ is now

$$
h_{1}=h-h_{x}=h-\int_{1}^{x} \frac{n^{2}(V+U)^{2} d x}{R^{4 / 3}},
$$

where $h$ is the height near the outlet, $h_{1}$ the height of 


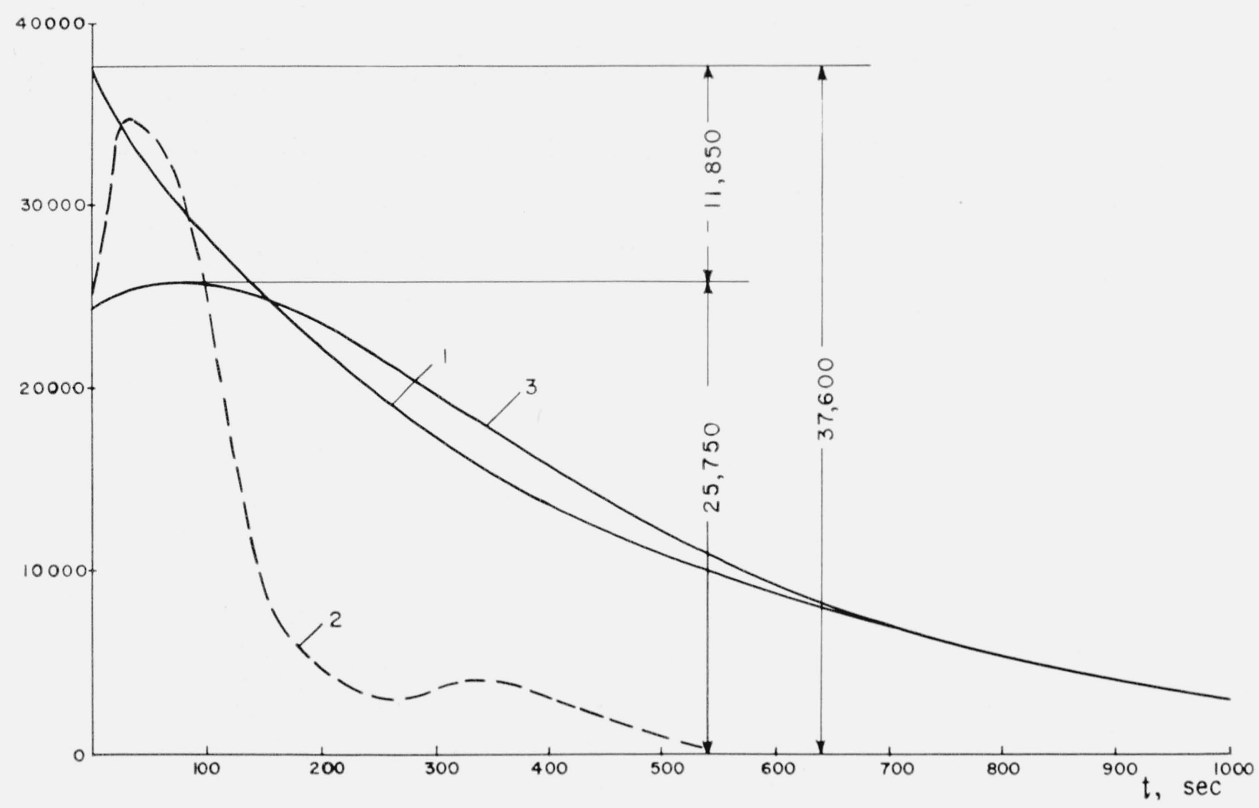

Figure 11. Three hydrographs of the Savage River Reservoir for the rectangular opening 250 $m$ by $18.5 \mathrm{~m}: 1$-first approximation hydrograph, when the movement of all waves is neglected; 2-fictitious inflow hydrograph, when the water accelerated by the movement of steep negative wave is considered as the inflow; 3-second approximation hydrograph, when the movement of steep negative wave is taken into account.

wave at section $x$, and $h_{x}$ the total resistance head from the outlet to the section $x$. Since $U$ and $R$ are the functions of $h_{1}$, the solution has to be made by successive approximations. As $h_{1}$ changes slowly, it is often sufficient to start with $h=$ constant for the computation of $U$ and $R$, and then correct values so obtained by successive approximations.

In fact, the potential energy of the steep negative wave is not all transformed into kinetic energy of accelerated water, one part of it being left as potential energy to be used as the flow resistance head.

The integration of the last term of eq (24) along the reservoir from the outlet to the place where $C-V=0$ would give the total resistance head. The volume $S_{r}$ which is not accelerated by the wave because of the resistance is part of the total volume $S_{h}$ (fig. 10) corresponding to wavefront height $h$, and is

$$
S_{r}=\int_{1}^{x_{0}} B_{x} h_{x} d x
$$

where $x_{0}$ is the place where $C-V=0$ and $B_{x}$ is the mean width of the area element for total resistance head $h_{x}$ at the section $x$.

The analytical integration of eqs (24) and (25) is not tractable even in simple cases like the pyramidal reservoir of rectangular cross section, figure 9, because the values $U$ and $R$ for a section $x$ depend on the sum of previous $d h$, and because of the expression $R^{4 / 3}$.
The practical procedure would be to compute $U$ and $R$ based on constant value $h$ along the reservoir, and to compute the slope of eq (23). By integrating $h_{x}=\int_{1}^{x} \frac{d h}{d x} d x$ the function of total resistance head $\mathrm{h}_{x}$ along the reservoir is obtained. Then $h_{1}=h-h_{x}$ gives the effective head of wavefront. With these values of $h_{x}$ new values $U$ and $R$ are again computed, and eqs (23) and (24) can be obtained as a second approximation. Succeeding approximations can be made by continuing the same procedure.

Due to the fact that the total velocity $(V+U$ increases in the upstream direction while the accelerated discharge decreases, it is usually sufficient to compute $h_{x}$ to the second or third approximation.

With the new values $h_{1}=h-h_{x}=f(x)$ it is now possible to determine a more exact fictitious hydrograph $P_{w}$.

For the computed function $h_{x}=f(x)$ the width $B_{x}$ has to be determined since $B_{x}=f(x)$. The integration of eq (25) gives $S_{r}$. The product of $B_{x} h_{x}$ along the reservoir makes possible the approximate computation of the mean distance $f_{r}$ of total volume $S_{r}$ from the level $H_{0}-h$ :

$$
f_{r}=\frac{E_{\tau}}{\gamma S_{r}}=\frac{1}{2 S_{r}} \int_{1}^{x_{0}} B_{x} h_{x}^{2} d x,
$$

where $E_{r}$ is the potential energy corresponding to the volume $S_{r}$ and the resistance head and $\gamma$ is the unit weight of water. 
The energy of the steep negative wave (fig. 10) is

$$
E_{w}=\gamma f_{0} S_{h}
$$

where $\mathrm{S}_{h}$ is total volume of reservoir between depths $H_{0}-h$ and $H_{0}$, and $f_{0}$ is the distance from the center of gravity of $S_{h}$ to the $H_{0}-h$ (fig. 10).

The ratio of energies

$$
e=\frac{E_{r}}{E_{w}}=\frac{f_{r} S_{r}}{f_{0} S_{h}}
$$

can be taken as a measure of the flow resistance effect on the transformation of the front height of steep negative wave.

As $h_{x}$ increases somewhat faster than $B_{x}$ decreases in the upstream direction, $B_{x} h_{x}$ increases from the outlet upstream. The effect of flow resistance decreases the accelerated flow $P_{w}$, slower at its highest values near the outlet and faster at its lowest values at the upstream end of the reservoir. The fictitious inflow hydrograph will be now closer to a straight line. As a practical approximation for rapid computations this hydrograph can be taken as triangularshaped for a valley reservoir.

\subsection{Simplest Procedure for the Computation of the Steep Negative Wave Effect With Resistance Influence}

A simple procedure for the computation of the approximate free outflow hydrograph in this case would be:

(1) Compute $h$ and $Q_{0} ;(2)$ compute $A, A_{1}, f, C$, $V, U$ along the reservoir for constant $h$; (3) determine $h_{x}$ along the reservoir; (4) recompute all values under (2); (5) recompute $h_{x}$ and all values under (2) until a sufficient approximation of the true values is obtained; $(6)$ compute $B_{x}$ as $f(x)$; (7) compute $S_{r}$ for given $h_{x}$ and $B_{x}$ curves; (8) determine $S_{h}$ from the storage function; (9) determine the difference $S_{h}-S_{r}$; (10) determine the triangular shape of fictitious inflow hydrograph $P_{w}$ with the maximum discharge $Q_{0}$ and the total time of fictitious hydrograph.

$$
t_{0}=\frac{2\left(S_{h}-S_{r}\right)}{Q_{0}} .
$$

The sum of the original and of the fictitious inflow hydrograph gives the total inflow hydrograph. The new inflow hydrograph, the maximum discharge $Q_{0}$ at the new starting level $k$ (fig. 6 ), the outflow rating curve and the storage function are now sufficient elements for any suitable routing procedure, based on the continuity equation only, of obtaining the free outflow hydrograph.

If the routing procedure involves the use of both the continuity and dynamic equations for unsteady flow which is gradually varied, the computed fictitious inflow discharges $P_{w}$, with their positions in time and along the reservoir, and the corresponding reservoir depths $H_{0}-h_{1}$ for the same times and places are the boundary conditions to be introduced in those equations.

\section{Discussion}

The computed outflow hydrographs from valley reservoirs in the case of sudden water release can be only approximations of the true hydrographs. The shape and the resistance to flow of valley reservoirs as well as the water movement itself along them are usually very complex. The closer an approximated hydrograph is to the true hydrograph, the more work is involved in the computation. The digital computers will, in some cases, give the most accurate results. The use of computers, however, is justified only in cases where very reliable and sufficient records and data about the reservoir, outlet, and inflow are available and where the great precision of results and speed of computation make their use economically feasible. Therefore, the use of simple numerical or graphical procedures for the computation of approximate outflow hydrographs is often very convenient, sensible, and economically desirable.

The movement of the steep negative wave along the reservoir accelerates water in the reservoir. Many factors affect this acceleration so that the approximations of the true outflow hydrograph depend on the factors either taken into account or neglected. The accelerated discharges are considered here as a fictitious inflow hydrograph to be taken as given either when only the storage continuity equation $P-Q=d S / d T$ is used for routing procedure or when both the continuity and dynamic equations are applied. The degree of unsteadiness of water movement is the criterion for the use of only the continuity equation or of both it and the momentum equation for the routing. The greater the height of the steep negative wave in relation to the reservoir depths, the higher and more unsteady the inflows, and the greater the flow resistance, then the higher will be the degree of unsteadiness of flow motion, and the use of the two de Saint Venant equations with the computation involved will be more necessary. The development of expressions for the quantitative determination of this criterion is a special problem not treated here. A criterion for the effect of flow resistance on the movement of steep negative wave is given by eq (28). When the ratio $e$ is higher than a selected limit, the flow resistance effect has to be taken into account, regardless of the routing procedure.

The fact that the steep wave is a discontinuity, not suitable to be considered as an unsteady flow gradually varied, and the fact that the movement and transformation of steep negative wave is not affected by small downstream disturbances in the reservoir, make the treatment of sudden water release different from an ordinary routing procedure. The determination of the outflow hydrograph can be divided into two procedures: $(a)$ The effect of steep negative wave and of flow resistance on the water acceleration, combined with the inflow hydrograph during the period of wave movement; $(b)$ the routing procedures, based on the position in place and time of accelerated discharges and inflow discharges along the reservoir, as boundary conditions. 
As the maximum outflow discharge $Q_{0}$ in the case of sudden water release of relatively larger openings is significantly lower than the discharge $Q_{\max }$ which corresponds to the given reservoir level and opening with already accelerated water in the reservoir, the maximum tailwater level for $Q_{0}$ is much lower than for $Q_{\max }$, so that the free outflow hydrograph (outflows without tailwater effect) is much more common than one would expect.

Washington, D.C.

(Paper 63B2-13)

\section{References}

[1] M. J. Boussinesq, Essai sur la théorie des eaux courantes, Mémoires presentés à l'Academie des sciences de l'Institut de France [?] 23 (Paris, 1877).

[2] H. Favre, Etude théoretique et expérimentale des ondes de translation dans les canaux découverts (Dunod, Paris, 1935).

[3] V. M. Yevdjevich, Analytical integration of the differential equation for water storage, J. Research NBS 63B, $43(1959)$.

[4] I. W. Howe, Flow measurement (ch. 3, Engineering hydraulics, Hunter Rouse, ed., pp. 212-222, John Wiley \& Sons, Inc., New York, N.Y., 1950).

[5] C. E. Kindvater and R. W. Carter, Discharge characteristics of rectangular thin-plate weirs, J. Hydraulies Div., Proc. Am. Soc. Civil Engrs. (Dec. 1957) Paper 1453.

[6] F. V. Pohle, Motion of water due to breaking of a dam, and related problems, symposium on gravity waves, NBS Circ. 521 (1952). 